\title{
Sedentary Behavior and Health-Related Quality of Life across Meeting Physical Activity Guidelines
}

\author{
Gene L. Farren*, Tao Zhang \\ Department of Kinesiology, Health Promotion, and Recreation, University of North Texas, Denton, TX 76203, USA \\ *Corresponding author: Gene.Farren@unt.edu
}

\begin{abstract}
Sedentary behavior may not differ between individuals meeting and not meeting physical activity guidelines (PAGs); however, sedentary behavior has been associated with lower levels of health-related quality of life (HRQoL). The purpose of this study was to investigate the influence of sedentary behavior on HRQoL across young adults in three physical activity engagement groups (i.e., did not meet aerobic or muscle-strengthening PAGs; met either aerobic PAGs or muscle-strengthening PAGs; and met both aerobic and muscle-strengthening PAGs) using moderation analyses. Participants were 427 young adults $\left(M_{\text {age }}=19.15 \pm 0.77\right)$ from Southwestern U.S. public university. Participants completed an online survey assessing sedentary behavior, physical activity profile, and HRQOL. On average, participants who did not meet aerobic or muscle-strengthening PAGs reported the lowest physical and psychosocial HRQoL and highest screen-time sedentary behavior, while participants who met both aerobic and muscle-strengthening PAGs reported the highest physical and psychosocial HRQoL and lowest screen-time sedentary behavior. Moderation analyses indicated meeting both aerobic and muscle-strengthening PAGs significantly buffered the negative influence of screen-time sedentary behavior on physical HRQoL. Meanwhile, meeting only one of the PAGs (i.e., aerobic or muscle-strengthening) did not significantly buffer the negative influence of screen-time sedentary behavior on physical HRQoL. Considering the current trends of sedentary behavior, the current study provided support in promoting regularly engaging in both aerobic and muscle-strengthening activity. Moreover, it provided evidence supporting the position that interventions designed to improve HRQoL should include both aerobic and muscle-strengthening activity.
\end{abstract}

Keywords: aerobic activity, muscle-strengthening activity, screen-time, college students

Cite This Article: Gene L. Farren, and Tao Zhang, "Sedentary Behavior and Health-Related Quality of Life across Meeting Physical Activity Guidelines.” World Journal of Preventive Medicine, vol. 5, no. 1 (2017): 10-18. doi: 10.12691/jpm-5-1-2.

\section{Introduction}

Regular physical activity has many health benefits including enhanced cardiorespiratory and musculoskeletal health, improved functional ability, enhanced self-indices, and decreased anxiety and depressive symptoms [1]. Further, the physical and mental health benefits associated with regular physical activity have been linked to improvements in health-related quality of life (HRQoL) [2]. HRQoL focuses on the influence health status has on quality of life [3], and is defined as the perceived physical and psychosocial health or well-being [4]. To maximize the likelihood of improving health status by achieving the health benefits associated with physical activity, the U.S. federal government collaborated with leading health professionals to establish minimum activity standards pertaining to both activity type and activity intensity for youth, adults, and older adults, entitled the 2008 Physical Activity Guidelines (PAGs) for Americans [5]. Evidences behind the PAGs also prompted other national governments including the U.K. to establish similar guidelines [6]. Though fundamentally similar to previous activity recommendations, the 2008 PAGs take into account that aerobic and muscle-strengthening activity (also referred to as resistance activity) are separate entities that uniquely and additively benefit health [7,8,9]. For instance, Blair and colleagues [10] posit that individuals should engage in muscle-strengthening activity, in addition to aerobic activity, at least twice a week, in that this activity type promotes the maintenance of lean muscle mass, improves muscular strength and endurance, and preserves functioning, which, in turn, enables long-term regular participation in physical activities and promotes quality of life.

Although aerobic and muscle-strengthening activity are considered separate entities that uniquely and additively benefit health, their combined impact is seldom assessed within research measuring dose-response relations between activity engagement frequency and health-outcomes such as HRQoL $[11,12]$. In fact, the overwhelming majority of research assessing the benefits of physical activity focuses solely on frequencies of moderate-to-vigorous aerobic physical activity (MVPA; [13]. For instance, research that found adhering to physical activity recommendations was significantly related to an enhanced HRQoL only measured aerobic physical activity $[14,15,16]$. However, Hart [17] found that when adults reported they engaged in muscle-strengthening (measured as yes or no) activity in addition to at least 10 minutes of moderate and/or 
vigorous aerobic activity (measure as yes or no), they had a greatly likelihood of reporting good HRQoL. This finding indicates that regularly engaging in both aerobic and muscle-strengthening activity likely may exhibit additive health benefits, which, in turn, improves health status, which, in turn, promotes HRQoL.

Despite strong evidence supporting the importance of engaging in both types of activity, data from the 2011 Behavioral Risk Factor Surveillance System (BRFSS) survey revealed $31.2 \%$ of U.S. young adults did not meet aerobic or muscle-strengthening PAGs, 38.1\% met only one PAG (i.e., either aerobic or muscle-strengthening PAGs), and only $30.7 \%$ met both aerobic and musclestrengthening PAGs $[8,18]$. Though this data revealed $56.8 \%$ of U.S. young adults (18-24 years) met the aerobic PAGs and $44.1 \%$ met the muscle-strengthening PAGs, it also indicated approximately one-half $(46.0 \%)$ of regular aerobic exercisers and one-third (31.1\%) of regular muscle-strengthening exercisers are not regularly engaging in the other activity type. Deductively, data from the BRFSS demonstrates individuals seemingly fall into one of three activity engagement groups based on meeting or not meeting PAGs. That is, generally, individuals either do not meet either of the PAGs, meet one of the PAGs (i.e., either aerobic or muscle-strengthening), or meet both of the PAGs. Given that aerobic and muscle-strengthening activity uniquely and additively benefit health $[9,19]$, it is reasonable to assume these groups would, respectively, demonstrate a stepwise increase in score of a perceptive health measure such as a HRQoL measure. Moreover, since the health effects associated with physical activity originates though biological mechanisms that improve health status [20], it is also reasonable to assume the influence of a negative health-risk factor such as sedentary behavior could also demonstrate a stepwise decline in score of this same perceptive health measure [21].

Over the last couple of decades, societal and technological changes have substantially impacted frequencies of physical activity and sedentary behavior [22,23]. Research indicates the frequency of physical activity has declined from previous decades [24,25], and in contrast, the frequency of sedentary behavior has increased from previous decades [22,26]. These findings suggest the inverse association between sedentary behavior and physical activity would be strong; however, research has yet to definitively support this assumption [27]. Moreover, the consequences of sedentary behavior are thought to be independent of physical activity $[21,27,28,29,30]$. In fact, Craft and colleagues [31] indicated sedentary behavior (measured as time spent sitting) may not differ between individuals meeting and not meeting PAGs (aerobic), and Matthews and colleagues [30] revealed high levels of MVPA may not mitigate the health-risks associated with prolonged sitting, especially screen-time sitting. In addition, research indicates higher levels of sedentary behaviors are significantly associated with lower levels of HRQoL and reductions in sedentary behavior may significantly improve physical HRQoL [32,33].

Though the relationships between HRQoL and meeting PAGs and HRQoL and sedentary behavior are established, it is not currently known whether sedentary behavior affects the relationship between meeting PAGs and HRQoL. That is, researchers have yet to investigate the sedentary behavior and its influence on HRQoL across individuals with varying physical activity engagement such as those not meeting either PAGs, those only meeting one of the PAGs, or those meeting both PAGs [29]. While research has suggested sedentary behavior may not differ between these groups and physical activity and sedentary behavior are independent determinants of health-outcomes [21,31], it is not known whether the negative impact of sedentary behavior on HRQoL varies between these groups. Given that the frequency of sedentary behavior is considered to be higher than previous decades [22,26] and individuals generally fall into one of these three groups, improving the understanding of the dose-response relations among these variables is crucial for developing interventions to enhance HRQoL [29]. Thus, the purpose of this study was to investigate whether sedentary behavior had a different influence on both physical and psychosocial HRQoL across the three physical activity engagement groups (i.e., NO PAGs, ONE PAGs, and BOTH PAGs).

Based on the literature reviewed [17,21,32,33,34,35], it was first hypothesized sedentary behavior, especially screen-time sitting, would be significantly negatively associated to both physical and psychosocial HRQoL. Second, it was hypothesized sedentary behavior and physical and psychosocial HRQoL would be significantly associated with engagement groups $[15,17,36]$. Lastly, it was hypothesized the influence of sedentary behavior on physical and psychosocial HRQoL would demonstrate a stepwise decline based on engagement group. That is, sedentary behavior would have the most influence on physical and psychosocial HRQoL among individuals who did not meet aerobic or muscle-strengthening PAGs and the least influence on physical and psychosocial HRQoL among individuals who meet both aerobic and muscle-strengthening PAGs [16,17,32,35].

\section{Methods}

Ethical approval for this study was obtained from the Institutional Review Board of a public research university located in Southwestern United States. Data for this crosssectional study were obtained from an online survey that was distributed summer of 2013 via e-mail by the university's Division of Student Affairs. Survey items investigated sedentary behaviors, physical activity profile (i.e., amount, type, and intensity), and sub-scales of HRQoL. No identifiable information was collected, and all students who received the recruitment e-mail were provided an informed consent notice. As an incentive, all who completed the 54-item survey were entered into a raffle for a $\$ 10.00$ gift card that was personally funded by the researchers.

\subsection{Participants}

Participants were 427 college students who had visited their university's recreation center at least once in the previous six months. Single-visit, casual, and regular recreation center visitors were targeted as participants for the purpose of obtaining a sample of young adults with varying frequencies of aerobic and muscle-strengthening 
activity engagement. An a priori power analysis indicated 395 participants were needed to have $80 \%$ power for detecting a small sized effect when employing .05 criterion of statistical significance.

\subsection{Measurements}

\subsubsection{Demographics}

Demographics assessed were age, sex, classification (e.g., freshman), ethnicity, and college major. Sex, ethnicity, and college major were used to compare sample normality with the university's general population.

\subsubsection{Physical Activity Profile}

Aerobic activity was assessed using the 7-item International Physical Activity Questionnaire-short form (IPAQ-SF), which was developed by a groups of experts in 1998 [37]. One item addressed sitting behavior and six items addressed participants' light-, moderate-, and vigorous-intensity aerobic activity over the last seven days. Where, for each intensity, one item assessed days per week and one item assessed hours per day. To specifically assess aerobic activity, activity examples given to represent intensity (i.e., moderate physical activities like...) were in accordance to the CDC and ACSM aerobic activity intensity examples [38]. Responses to the four items addressing moderate- and vigorous-intensity aerobic activity were combined by first computing a total time for each intensity and then converting both intensities' total to a single MVPA temporal scale [38]. Then, total MVPA was then analyzed to determine if participants met or did not meet the aerobic PAGs (i.e., MVPA $\geq 150 \mathrm{~min}$. = met aerobic PAGs).

To assess muscle-strengthening activity, an additional item was created using the same IPAQ-SF format (i.e., During the last 7 days, how many days did you do muscle-strengthening activities like weight lifting, calisthenics, or other resistance exercises that worked all major muscle groups [legs, hips, back, abdomen, chest, shoulders, and arms]?). Previous research used similarly constructed items to assess muscle-strengthening activity $[39,40]$. According to the PAGs to be categorized as meeting muscle-strengthening PAGs, participants needed to report doing muscle-strengthening activities on two or more days during the last 7 days [38]. In the present study, scores of eight items were found to have high internal consistency $(\alpha=.92)$. After participants' aerobic and muscle-strengthening PAGs statuses were determined, a single categorical group variable was created (i.e., engagement) that labeled participants as 0 , 1, or 2 (i.e., NO PAGs, ONE PAGs, or BOTH PAGs, respectively).

\subsubsection{Sedentary Behavior}

The 18-item Sedentary Behavior Questionnaire (SBQ) [41] was used to assess students' sedentary activity. Students were asked to indicate the amount of time, on a 9-point temporal scale ranging from none (0) to 6 hours or more (8), they spent doing nine activities while sitting (i.e., watching television or videos, playing video games, listening to music, talking or texting on the phone, doing school/office work, reading a book or magazine, playing a musical instrument, doing arts and crafts, and driving/riding in a car, bus, or train) on a typical day. Responses to the first two items were summed to compute the screen-time sitting per day (ST), and then all other items were summed to compute the non-screen-time sitting per day (non-ST). Weekday and weekend day were assessed separately using the same nine items, and weekday and weekend day were multiplied by the appropriate days per week and then summed to obtain 7-day averages [42]. Rosenberg and colleagues [41] found the SBQ had moderate to excellent test-retest reliability for the nine activities and for the total scale on weekdays and weekend days. For the current study, validity was supported by correlation with the IPAQ-SF sitting-time item $(r=.45, p<.01)$.

\subsubsection{Health-Related Quality of Life}

The 23-item Pediatric Quality of Life Inventory ${ }^{\mathrm{TM}}$ Generic Core Scales Young Adult Version was used to evaluate participants' self-reported HRQoL [43]. Participants were asked to indicate how much of a problem each item had been during the past seven days, on a 5-point scale ranging from never (0) to always (4). To designate higher scores with a higher HRQoL, responses to HRQoL items were reversed scored and linearly transformed to a 0-100 scale $[43,44]$. This HRQoL measure yields four subscale factors (i.e., physical, emotional, social, and school functioning). Besides the physical factor, for the current study, a higher-order factor, psychosocial, was computed by summing the latter three subscale factors [43]. Previous research found scores for this measure had high internal consistency ( $\alpha=.89$ ) in young adults [45]. Scores for all HRQoL factors in the present study had high internal consistency ( $\alpha=.87$ to .95$)$.

\subsection{Statistical Analyses}

Statistical analyses were conducted using SPSS ${ }^{\circledR} 22.0$ (IBM Corporation, Armonk, NY, USA) for Windows ${ }^{\circledR} /$ Apple $\mathrm{Mac}^{\circledR}$, and statistical significance was set at $\alpha<.05$. $G^{*}$ Power Version 3.1.9.2 [46] was used to perform an a priori power analyses. After all studied variables were computed missing values, univariate outliers, and multivariate outliers and distributions were explored. Missing data analysis revealed 21 participants were missing a significant amount of data (i.e., missing an entire scale or multiple scales). Little's MCAR test indicated these participants' data were not missing completely at random, so they were excluded [47]. Two participants were discovered to have univariate outliers [48]. Based on Mahalanobis, Cook’s, leverage distances three participants were discovered to have multivariate outliers $[49,50]$. Once missing data and outliers were excluded ( $n=26)$, score reliabilities and descriptive statistics were assessed, and correlation analyses were conducted. A paired-samples $t$-test was conducted to explore mean differences between week day and weekend day sitting behaviors. Independent-samples t-tests and chi-squared tests were conducted to explore mean differences and associations between sexes in order to determine if sex should be controlled. A one-way ANOVA was conducted to examine sitting and physical and psychosocial HRQoL mean differences among engagement groups. Lastly, two moderation analyses were conducted 
using the PROCESS macro for SPSS ${ }^{\circledR}$ [51] to assess whether the influence of sitting on physical HRQoL (Model 1) and psychosocial HRQoL (Model 2) were different across the engagement groups. The meeting NO PAGs group $(0,0)$ served as the reference or indicator group. Thus, the significance test of the sedentary behavior variable (i.e., ST and non-ST) represented the significance test of the simple slope for this group [44,51].

\section{Results}

\subsection{Descriptive Statistics}

Descriptive statistics revealed participants $(N=401)$ had a mean age of $19.15 \pm 0.77$ years; were $53.1 \%$ male; were $50.4 \%$ sophomores, $45.6 \%$ freshman, and $4 \%$ juniors; were $45.6 \%$ 56.4\% White, $17.5 \%$ African-American, 11.5\% Hispanic/Latino, 6.5\% Asian, and 8.1\% other; and represented 41 different college majors. Demographic data were comparable to the university's general population [52]. Regarding sedentary behavior or sitting-time, a paired-samples $t$-test revealed weekday ST and non-ST were not significantly different from weekend day ST and non-ST $(t=-1.09, p=.07$ and $t=0.89, p=.37$, respectively). Therefore, ST and non-ST values reflect seven day averages in further analyses. Descriptive statistics indicated participants spent, on average, $7.66 \pm 3.94$ $\mathrm{hr}$ /day sitting with $3.11 \pm 1.17 \mathrm{hr} /$ day classified as ST. Regarding aerobic and muscle-strengthening data, descriptive statistics for the revealed approximately $34.9 \%$ of participants did not meet aerobic or muscle-strengthening PAGs, 34.2\% met either aerobic or muscle-strengthening PAGs, and $30.9 \%$ of participants met both aerobic and muscle-strengthening PAGs. Further, 56.1\% of participants met aerobic PAGs (i.e., reported at least 150 minutes of MVPA) and 39.9\% met muscle-strengthening PAGs (i.e., reported doing muscle-strengthening activities on two or more days). Specifically, participants reported an average of $25.50 \pm 18.26 \mathrm{~min} /$ day of MVPA and $2.31 \pm 1.67$ day/week of muscle-strengthening activity. Lastly, descriptive statistics indicated the average physical HRQoL was $81.43 \pm 15.98$, psychosocial HRQoL was $74.89 \pm 16.94$, and total HRQoL was 77.16 \pm 15.67 .

Table 1. Means, Standard Deviations, and Correlation Coefficients for the Sitting, Engagement, and HRQoL Variables

\begin{tabular}{|c|c|c|c|c|c|c|c|c|c|c|}
\hline Variable & 1. & 2. & 3. & 4. & 5. & 6. & 7. & 8. & 9. & 10. \\
\hline 1. Non-screen-time sitting & - & $.64^{* *}$ & $.97^{* *}$ & $-.12^{*}$ & $-.14^{* *}$ & $-.14^{* *}$ & .06 & -.04 & .03 & .01 \\
\hline 2. Screen-time sitting & & - & $.80^{* *}$ & $-.17^{* *}$ & $-.13^{* *}$ & $-.15^{* *}$ & $-.15^{* *}$ & $.14^{* *}$ & -.01 & $-.14^{* *}$ \\
\hline 3. Total time sitting & & & - & $-.15^{* *}$ & $-.14^{* *}$ & $-.15^{* *}$ & -.00 & .01 & .02 & -.03 \\
\hline 4. Physical HRQoL & & & & - & $.76^{* *}$ & $.89^{* *}$ & $.52^{* *}$ & $-.51^{* *}$ & $.11^{*}$ & $.40^{* *}$ \\
\hline 5. Psychosocial HRQoL & & & & & - & $.97^{* *}$ & $.41^{* *}$ & $-.37^{* *}$ & .03 & $.35^{* *}$ \\
\hline 6. Total HRQoL & & & & & & - & $.48^{* *}$ & $-.44^{* *}$ & .06 & $.39^{* *}$ \\
\hline 7. Engagement & & & & & & & - & $-.84^{* *}$ & -.01 & $.86^{* *}$ \\
\hline 8. NO PAGs ${ }^{\mathrm{a}}$ & & & & & & & & - & $-.53^{* *}$ & $-.47^{* *}$ \\
\hline 9. ONE PAGs ${ }^{\mathrm{a}}$ & & & & & & & & & - & $-.50^{* *}$ \\
\hline 10. BOTH PAGs ${ }^{\mathrm{a}}$ & & & & & & & & & & - \\
\hline$M$ & 4.54 & 3.11 & 7.66 & 81.43 & 74.89 & 77.16 & 0.96 & $n=140$ & $n=137$ & $n=124$ \\
\hline$S D$ & 3.09 & 1.17 & 3.94 & 15.98 & 16.94 & 15.67 & 0.81 & $34.9 \%$ & $34.2 \%$ & $30.9 \%$ \\
\hline
\end{tabular}

Note. $N=401$. Sitting values in hr/day. Engagement $=$ PAGs adherence status, $0=$ NO PAGs, $1=$ ONE PAGs, and $2=$ BOTH PAGs.

${ }^{\mathrm{a}}$ Dummy variables representing the three engagement groups with $0=$ no and $1=$ yes.

${ }^{*} p<.05 ;{ }^{* *} p<.01$.

Table 2. Results of Independent-Samples t-tests for Sedentary Behavior and Health-Related Quality of Life (HRQoL) Variables by Sex

\begin{tabular}{|c|c|c|c|c|c|c|c|c|}
\hline \multirow[b]{3}{*}{ Variable } & \multicolumn{4}{|c|}{ Sex } & \multirow{3}{*}{$\begin{array}{c}\text { 95\% CI for } \\
\text { Mean } \\
\text { Differences }\end{array}$} & \multirow[b]{3}{*}{$t$} & \multirow[b]{3}{*}{$d f$} & \multirow[b]{3}{*}{$p$-value } \\
\hline & \multicolumn{2}{|c|}{ Female $(n=188)$} & \multicolumn{2}{|c|}{ Male $(n=213)$} & & & & \\
\hline & $M$ & $S D$ & $M$ & $S D$ & & & & \\
\hline Non-screen-time sitting & 4.45 & 2.83 & 4.63 & 3.31 & $-0.78,0.42$ & -0.59 & 398.65 & .55 \\
\hline Screen-time sitting & 3.02 & 1.23 & 3.20 & 1.11 & $-0.41,0.05$ & -1.63 & 399 & .13 \\
\hline Physical HRQoL & 80.12 & 15.94 & 82.58 & 15.96 & $-5.59,0.68$ & -1.60 & 399 & .13 \\
\hline Psychosocial HRQoL & 74.51 & 15.58 & 75.22 & 18.08 & $-4.01,2.59$ & -0.57 & 398.77 & .67 \\
\hline
\end{tabular}

Note. $N=401$. Sitting values in hr/day.

Table 3. Results of the One-way ANOVA and Tukey's HSD Post Hoc Tests

\begin{tabular}{|c|c|c|c|c|c|c|c|c|c|c|}
\hline \multirow[b]{2}{*}{ Variable } & \multicolumn{2}{|c|}{$\begin{array}{l}\text { 1. NO PAGs } \\
(n=140)\end{array}$} & \multicolumn{2}{|c|}{$\begin{array}{l}\text { 2. ONE PAGs } \\
(n=137)\end{array}$} & \multicolumn{2}{|c|}{$\begin{array}{l}\text { 3. BOTH PAGs } \\
(n=124)\end{array}$} & \multirow[b]{2}{*}{$F(2,397)$} & \multirow[b]{2}{*}{$p$-value } & \multirow[b]{2}{*}{$\eta^{2}$} & \multirow[b]{2}{*}{ Tukey's HSD } \\
\hline & $M$ & $S D$ & $M$ & $S D$ & $M$ & $S D$ & & & & \\
\hline Non-screen-time sitting & 4.23 & 2.57 & 4.78 & 3.46 & 4.63 & 3.18 & 1.19 & .31 & .01 & - \\
\hline Screen-time sitting & 3.31 & 0.89 & 3.13 & 1.29 & 2.87 & 1.27 & 4.60 & $<.05$ & .02 & $1>3$ \\
\hline Physical HRQoL & 70.45 & 14.41 & 84.16 & 15.30 & 90.80 & 10.13 & 78.06 & $<.01$ & .28 & $1<2<3$ \\
\hline
\end{tabular}

Note. $N=401$. Sitting values in hr/day.

${ }^{a}$ Only statistically significant differences are noted $(p<.01)$. 


\subsection{Variable Associations}

Table 1 presents the means, standard deviations, and correlation coefficients of the sitting, engagement, and HRQoL variables. Correlation coefficients revealed ST was significantly inversely related to engagement and physical and psychosocial HRQoL. Though non-ST was significantly inversely related to physical and psychosocial HRQoL, it was not significantly associated to engagement or the dummy variables representing the three engagement groups. Correlation coefficients also indicated physical and psychosocial HRQoL were significantly associated to engagement and the dummy variables representing the three engagement groups. Specifically, point-biserial coefficients indicated physical HRQoL was inversely associated to NO PAGs and directly associated to ONE PAGs and BOTH PAGs. Whereas, psychosocial HRQoL was also inversely associated to NO PAGs and directly associated to BOTH PAGs, but not significantly associated to ONE PAGs.

Independent-samples $t$-test revealed values for non-ST, ST, and physical and psychosocial HRQoL were not statistically significantly different between sexes (see Table 2). A chi-squared test of independence indicated sex and engagement group were not significantly associated, $\chi^{2}=4.82, p=.09$. Given the results of the previous tests sex was not controlled in further analyses. The one-way ANOVA indicated there were statistically significant differences in mean ST and mean physical and psychosocial HRQoL between the three engagement groups. Specifically, post hoc analyses indicated the NO PAGs group, on average, had significantly greater ST than the BOTH PAGs group. In addition, the BOTH PAGs group, on average, had significantly higher physical and psychosocial HRQoL than the ONE PAGs group, and the ONE PAGs group, on average, had significantly higher physical and psychosocial HRQoL than the NO PAGs group. Results of the one-way ANOVA including post hoc analyses using Tukey's HSD post hoc criterion for significance are presented in Table 3.

\subsection{Moderation Analyses}

Summaries of the moderation analyses for Models 1 and 2 are presented in Table 4 . Model 1 yielded a statistically significant result, $R^{2}=.30, F(5,395)=38.90$, $p<.01$. The increase in variance explained due to the interaction was also statistically significant, $\Delta R^{2}=.01$, $F(2,395)=3.46, p<.05$. Regression coefficients indicated the average estimated physical HRQoL in the NO PAGs group, at mean ST, was 71.07. In contrast, at mean ST, the average estimated physical HRQoL was 13.12 units higher $(p<.01)$ in the ONE PAGs group, and 19.83 units higher $(p<.01)$ in the BOTH PAGs group. Regarding slope, the average estimated deviation in physical HRQoL per additional hour of ST in the NO PAGs group was statistically significantly different from zero $(B=-3.12, p<.05)$. That is, physical HRQoL in the NO PAGs group decreased by an average of 3.12 units per additional hour of ST. In contrast, the average estimated deviation in physical HRQoL per additional hour of ST in the ONE PAGs group was not statistically significantly different from slope in the NO PAGs group $(B=1.11, p=.50)$. In addition, the regression coefficient representing the conditional effect of ST indicated the average estimated deviation in physical HRQoL per additional hour of ST in the ONE PAGs group was statistically significantly different from zero $(B=-2.01, p<.05)$. Lastly, the average estimated deviation in physical HRQoL per additional hour of ST in the BOTH PAGs group was statistically significantly different from slope in the NO PAGs group $(B=3.54$, $p<.05)$, and the regression coefficient representing the conditional effect of ST indicated the slope in the BOTH PAGs was not significantly different than zero $(B=0.42$, $p=.60)$.

Table 4. Summary of the Moderation Analyses for Model 1 and Model 2

\begin{tabular}{|c|c|c|c|c|c|c|c|c|c|}
\hline \multirow[b]{2}{*}{ Model } & \multirow[b]{2}{*}{$R^{2}$} & \multirow[b]{2}{*}{$F(5,395)$} & \multirow[b]{2}{*}{$B$} & \multirow[b]{2}{*}{$S E$} & \multirow[b]{2}{*}{$t$} & \multirow[b]{2}{*}{$p$-value } & \multicolumn{3}{|c|}{ Conditional Effect $^{\mathrm{a}}$} \\
\hline & & & & & & & $B$ & $S E$ & $p$-value \\
\hline 1. Physical HRQoL & $.30^{\mathrm{b}}$ & 38.90 & & & & $<.01$ & & & \\
\hline ONE PAGs (D1) & & & 13.12 & 1.79 & 7.33 & $<.01$ & & & \\
\hline BOTH PAGs (D2) & & & 19.83 & 1.52 & 13.04 & $<.01$ & & & \\
\hline Screen-time sitting (ST) & & & -3.12 & 1.39 & -2.24 & $<.05$ & -3.12 & 1.39 & $<.05$ \\
\hline ST*ONE PAGs & & & 1.11 & 1.63 & 0.68 & $=.50$ & -2.01 & 0.85 & $<.05$ \\
\hline ST*BOTH PAGs & & & 3.54 & 1.60 & 2.21 & $<.05$ & 0.42 & 0.80 & $=.60$ \\
\hline 2. Psychosocial HRQoL & $.18^{\mathrm{c}}$ & 21.69 & & & & $<.01$ & & & \\
\hline Constant & & & 66.86 & 1.13 & 59.14 & $<.01$ & & & \\
\hline ONE PAGs (D1) & & & 9.09 & 1.78 & 5.10 & $<.01$ & & & \\
\hline $\mathrm{ST}$ & & & -3.17 & 1.22 & -2.60 & $<.05$ & -3.17 & 1.22 & $<.05$ \\
\hline ST*ONE PAGs & & & 2.23 & 1.55 & 1.44 & $=.15$ & -0.93 & 0.96 & $=.33$ \\
\hline ST*BOTH PAGs & & & 3.35 & 2.02 & 1.66 & $=.10$ & 0.19 & 1.61 & $=.91$ \\
\hline
\end{tabular}

Note. $N=401$. D1 = dummy variable 1 ; D2 = dummy variable 2 .

${ }^{\mathrm{a}}$ The effect of ST in groups defined by engagement group.

${ }^{\mathrm{b}} \Delta R^{2}=.01, F(2,395)=3.46, p<.05$

${ }^{\mathrm{c}} \Delta R^{2}=.01, F(2,395)=1.65, p=.19$. 


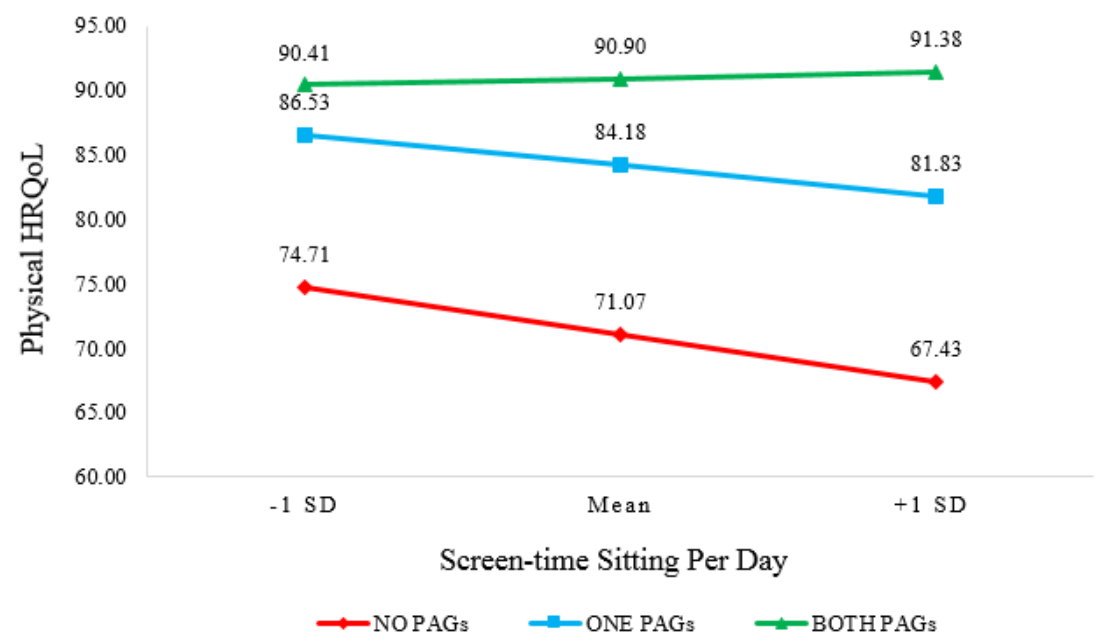

Figure 1. Line graphs illustrating the influence of screen-time sitting $(M=3.12 \pm 1.16)$ on physical health-related quality of life $(\mathrm{HRQoL})$ across three physical activity engagement groups

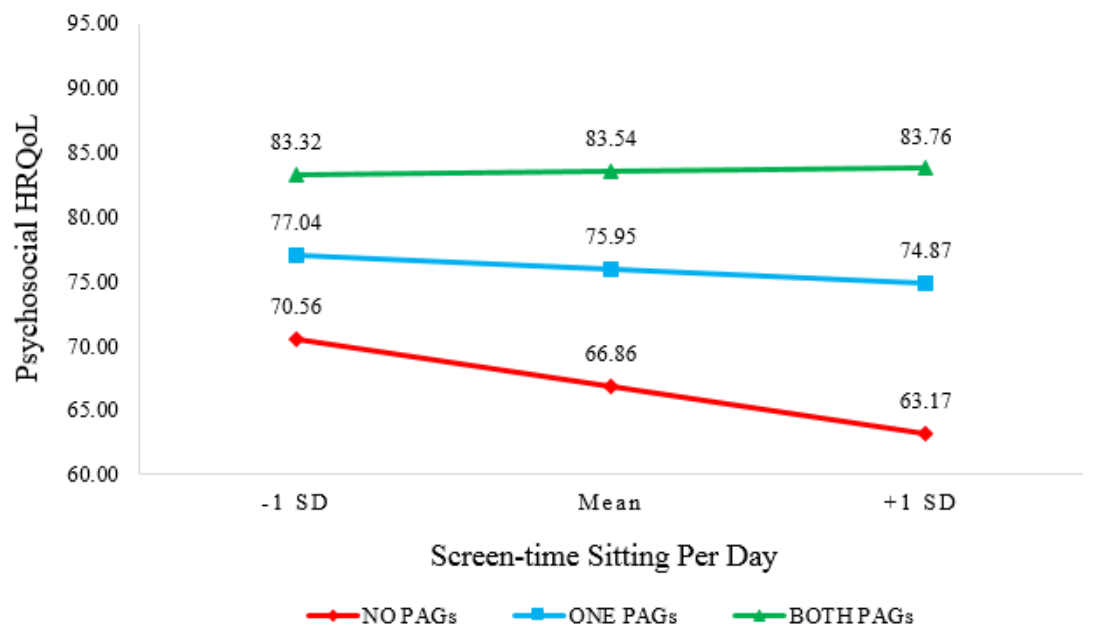

Figure 2. Line graphs illustrating the influence of screen-time sitting $(M=3.12 \pm 1.16)$ on psychosocial HRQoL across three physical activity engagement groups

Model 2 also yielded a statistically significant result, $R^{2}=.28, F(5,395)=21.69, p<.01$, but the increase in variance explained due to the interaction was not statistically significant, $\Delta R^{2}=.01, F(2,395)=1.65$, $p=.19$. Regression coefficients revealed the average estimated psychosocial HRQoL in the NO PAGs group, at mean ST, was 66.86. In contrast, at mean ST, the ONE PAGs group had an average estimated psychosocial HRQoL that was 9.09 units higher $(p<.01)$ than the NO PAGs group, and the BOTH PAGs group had an average estimated psychosocial HRQoL that was 16.68 units higher $(p<.01)$ than the NO PAGs group. Regarding slope, the average estimated deviation in psychosocial HRQoL per additional hour of ST in the NO PAGs group was statistically significantly different from zero $(B=$ 3.17, $p<.05)$. Thus, psychosocial HRQoL in the NO PAGs group decreased by an average of 3.17 units per additional hour of ST. In contrast, the average estimated deviation in psychosocial HRQoL per additional hour of ST in the ONE PAGs and BOTH PAGs groups were not statistically significantly different from slope in the NO PAGs group $(B=2.23, p=.15$ and $B=3.35, p=.10$, respectively). However, the regression coefficients representing the conditional effect of ST indicated the average estimated deviation in psychosocial HRQoL per additional hour of ST in the ONE PAGs and BOTH PAGs groups were not statistically significantly different from zero $(B=-0.93, p=.33$ and $B=0.19, p=.91)$. To illustrate the two aforementioned models, two line graphs were created (see Figure 1 and Figure 2). Simple slope equations examining the influence of low ST (i.e., one standard deviation below the mean), mean ST, and high ST (i.e., one standard deviation above the mean) on physical (a) and psychosocial (b) HRQoL across the three different engagement groups were computed [53,54,55]. Resulted values from these computations were plotted in Figure 1 and Figure 2.

\section{Discussion}

The present study examined influence of sedentary behavior on physical and psychosocial HRQoL across groups based on adherence to PAGs. Similar to previous research the sedentary behavior remained rather stable between weekday and weekend days [56,57,58]. In addition, the values for time spent sitting in the current study fell within values found in previous research assessing similar relations in young adults [35,59], and physical activity engagement group distribution in the 
current study was congruent to the distribution found in the 2011 BRFSS data $[8,18]$. Further, HRQoL scores were also consistent with previous research assessing similar relations in young adults [45]. Collectively, these consistencies support the generalizability of the findings in the current study.

Consistent with previous literature, correlation analyses revealed physical and psychosocial HRQoL were both significantly inversely associated ST [17,21,32,33,34,35] and directly associated to physical activity engagement $[15,17,36]$, which supported the first and second hypotheses, respectively. Though meeting PAGs (i.e., engagement) was significantly associated to ST, it was not significantly associated to non-ST and more specifically total time sitting, which was consistent to previous research that found equivalent sitting-time between those meeting and not meeting PAGs [31]. On the other hand, given that ST was significantly associated with engagement, the position that the influence of sedentary behavior on health and health determinants varies based on the type of sedentary behavior was further supported [60]. Congruent with previous research [16,17], engagement and physical and psychosocial HRQoL did not significantly vary between sexes. Though males did, on average, report higher levels of sedentary behavior (i.e., ST and non-ST) than females, levels of sedentary behavior were not significantly different between sexes, which was incongruent with previous research [59,61]. This incongruent finding suggests further research is needed to examine the sedentary habits between sexes in young adults. While, in part, further supporting the second hypothesis, the oneway ANOVA also demonstrated a stepwise decease in ST and a stepwise increase in HRQoL with respect to engagement, which demonstrated the need to further explore interaction effects among these variables.

Consistent with previous research $[16,17,32,35]$, the NO PAGs group, on average, reported the lowest physical and psychosocial HRQoL at all three levels of ST (i.e., low, mean, and high), and the BOTH PAGs group, on average, reported the highest physical and psychosocial HRQoL at all three levels of ST. These findings effectively demonstrate how the health benefits associated with meeting PAGs positively impact HRQoL. Moreover, Model 1 revealed a statistically significant interaction effect, in that, on average, meeting both aerobic and muscle-strengthening PAGs significantly buffered the influence of ST on physical HRQoL (see Figure 1). Though meeting only one of the PAGs was associated with a higher physical HRQoL than not meeting any PAGs, it did not, on average, significantly buffer the influence of ST on physical HRQoL. On the other hand, while the buffering was not statistically significant, this result still demonstrated a stepwise decline of the influence of ST on physical HRQoL and further supported meeting PAGs as a positive health determinate. As for Model 2, a statistically significant interaction effect was not observed. However, as shown in Figure 2 and Table 4, Model 2 did reveal not meeting either of the PAGs was associated with an average estimated influence of ST on psychosocial HRQoL that was statistically significantly different from zero, while meeting one of the PAGs and/or meeting both PAGs was associated with average estimated influence of ST of psychosocial HRQoL that was not statistically significantly different from zero. In addition, though not statistically significantly, Model 2 demonstrated a stepwise decline of the influence of ST on psychosocial HRQoL likely existed. Overall, both Model 1 and Model 2 indicated sedentary behavior had the least influence on physical and psychosocial HRQoL among individuals who met both PAGs, which supported the third hypothesis.

Although previous research indicated the interaction between sedentary behavior and MVPA on HRQoL only approached significance [35] in adults, results of the current study indicated interaction between ST and physical activity engagement (i.e., meeting both aerobic and muscle-strengthening PAGs) on physical HRQoL was statistically significant in young adults. That is, while the interaction between sedentary behavior and meeting only one PAGs was not statistically significant, which was consistent with previous research [35], the interaction between sedentary behavior and meeting both PAGs was statistically significant. This result might be best supported by previous research that indicated meeting both aerobic and muscle-strengthening PAGs had additive health benefits over and above the health benefits associated with meeting only one of the PAGs [17,39,62].

To our knowledge, this was the first study to examine the influence of sedentary behavior on physical and psychosocial HRQoL across young adults with varying physical activity engagement. Therefore, this study expands the existing knowledge regarding the relations among sedentary behavior, aerobic and musclestrengthening activity engagement, and HRQoL in young adults. Though the scope of the negative healthconsequences of sedentary behavior has yet been fully discovered [30], results of the current study support the concept that regularly engaging in physical activity, especially regularly engaging in both aerobic and musclestrengthening activity, may, in part, mitigate the negative influence of health-risk factors such as sedentary behavior on health-outcomes such as HRQoL in young adults. Considering the prevalence of sedentary behavior in young adults $[22,26]$, these results provided additionally evidence for the importance of meeting PAGs, in that, on average, meeting one and/or both PAGs was associated with higher physical and psychosocial HRQoL and lower levels of sedentary behavior. Moreover, meeting both aerobic and muscle-strengthening PAGs significantly buffered the influence of sedentary behavior on physical HRQoL.

On the other hand, the findings of the current study should be considered in light of the following limitations. First, the particulars of this sample may limit the generalizability of these results to similar samples. However, considering the sedentary behavior, activity profile, and HRQoL values were similar to the values found in previous studies $[8,18,35,45,59]$, it appears the present sample adequately represented young adults in the U.S. To increase generalizability, subsequent research should examine samples from different populations such as young adults from different universities, countries, and learning settings, as well as different age groups to determine whether findings remain consistent. Second, though self-report measures allow for easier dissemination and implementation, past research has indicated direct 
measures are more accurate, reliable, and valid [63,64]. In addition, self-report data are subject to social desirability biases, which might have, according to previous research, underestimated and overestimated students' responses $[2,65]$. Therefore, future researchers are advised to further examine the principal findings of the current study by using direct measures for assessing sedentary behavior, physical activity, and HRQoL such as pedometers, accelerometers, observations, physiological health factors, and health records. Third, cross-sectional design does not allow researchers to establish causality or observe change. Thus, to investigate whether these relationships are causal or change over time, future researchers should utilize longitudinal research design [54].

Other possible extraneous variables for the current study might be selection bias, in that participants were not randomly selected. Therefore, the data obtained in this sample may be more representative of individuals who volunteered due to having a vested interest in its context. Also, the surveys were online, thus, students did not have the opportunity to ask clarifying questions during assessment, which could lead to measurement error due to unintended or incorrect responses [66].

\section{Conclusion}

Considering the current trends of sedentary behavior, physical activity, and negative health-outcomes, improving our understanding of the dose-response relations among these variables is becoming more and more pertinent. Accurately determining the influence of sedentary behavior on health-related outcomes such as HRQoL within different types and doses of physical activity could help in developing interventions to promote our general well-being. That is, an improved understanding of the varying influences of sedentary behavior and physical activity on health-outcomes could help us manage and prevent the onset of many negative health-consequences for future generations. As such, in order to offset the potential negative consequences of sedentary behavior that may be inevitable considering the current societal and technological changes, this study indicated interventions designed to improve HRQoL should include multiple types of physical activity rather than a single type. In addition, future research studies should examine the influence of sedentary behavior on multiple health-related outcomes across different types and doses of physical activity within multiple populations, while also using objective measurements in order to more precisely identify how sedentary behavior influences our health.

\section{Acknowledgements}

We would first like to thank the students for volunteering to participate in this investigation. Second, we would like to give a special thanks to the university's Assistant Vice President for Student Affairs and Director of Recreational Sports. Their collaboration was essential for distributing our survey, and they graciously gave their time and supplied us with information that gave purpose and possibility to our study.

\section{References}

[1] Centers for Disease Control and Prevention. Physical activity and health: The benefits of physical activity. Available at: http://www.cdc.gov/physicalactivity/basics/pa-health/. Accessed July 15, 2015.

[2] Anokye NK, Trueman P, Green C, et al. Physical activity and health related quality of life. BMC Public Health 2012;12:624-31.

[3] Healthy People 2020. Health-related quality of life and well-being. 2010.

[4] Centers for Disease Control and Prevention. Health-related quality of life (HRQOL). Available at:

https://www.cdc.gov/hrqol/index.htm. AccessedJune 1, 2016.

[5] Physical Activity Guidelines Advisory Committee. Physical Activity Guidelines Advisory Committee Report, 2008. Washington, DC: U.S. Department of Health and Human Services; 2008.

[6] Bull FC, The Expert Working Groups. Physical activity guidelines in the U.K.: Review and recommendations. School of Sport, Exercise and Health Sciences, Loughborough University: 2010.

[7] Loustalot F, Carlson SA, Kruger J, et al. Muscle-strengthening activities and participation among adults in the United States. Res Q Exerc Sport 2013;84:30-8.

[8] Centers for Disease Control and Prevention. Adult participation in aerobic and muscle-strengthening physical activities-United States, 2011. Morb Mortal Wkly Rep 2013;62:326-30.

[9] Haskell WL, Lee IM, Pate RR, et al. Physical activity and public health: Updated recommendation for adults from the American College of Sports Medicine and the American Heart Association. Med Sci Sport Exerc 2007;39:1423-34.

[10] Blair SN, Lamonte MJ, Nichaman MZ. The evolution of physical activity recomendations: How much is enough? Am J Clin Nutr 2004;79(suppl):913S-20S

[11] Morrow JR, Vingren JL. But wait-the PA guidelines include muscle-strengthening activities, too! J Appl Physiol 2011; 111: 609-10.

[12] Oja P. Dose response between total volume of physical activity and health and fitness. Med Sci Sport Exerc 2001; 33: S428-37.

[13] Carnethon MR. Physical activity and cardiovascular disease: How much is enough? Am J Lifestyle Med 2009;3:44S-49S.

[14] Vuillemin A, Boini S, Bertrais S, et al. Leisure time physical activity and health-related quality of life. Prev Med (Baltim) 2005; 41: 562-9.

[15] Austin S, Qu H, Shewchuk RM. Association between adherence to physical activity guidelines and health-related quality of life among individuals with physician-diagnosed arthritis. Qual Life Res 2012; 21: 1347-57.

[16] Hart PD. Meeting recommended levels of physical activity and health-related of life in rural adults. J Lifestyle Med 2016; 6: 1-6.

[17] Hart PD. Muscle strengthening activity in addition to physical activity and health-related quality of life in adults. World J Prev Medincine 2015;3:24-7.

[18] Centers for Disease Control and Prevention. Behavioral Risk Factor Surveillance System. Available at: http://www.cdc.gov/brfss/annual_data/annual_2011.htm. AccessedDecember 10, 2014.

[19] Centers for Disease Control and Prevention. Trends in the prevalence of physical activity and sedentary behaviors; National YRBS: 1991-2013. Atlanta, GA: 2014.

[20] Hamilton MT, Hamilton DG, Zderic T. Role of low energy expenditure and sitting in obesity, metabolic syndrome, type 2 diabetes, and cardiovascular disease. Diabetes 2007;56:2655-67.

[21] de Rezende LFM, Lopes MR, Rey-Ló Pez JP, et al. Sedentary behavior and health outcomes: An overview of systematic reviews. PLoS One 2014;9:e10562.

[22] Brownson RC, Boehmer TK, Luke DA. Decling rates of physical activity in the United States: What are the contributors? Annu Rev Public Health 2005;26:421-43.

[23] Bassett DR, John D, Conger SA, et al. Trends in Physical Activity and Sedentary Behaviors of United States Youth. J Phys Act Heal 2015;12:1102-11 
[24] National Center for Health Statistics. Health, United States, 2015: With special feature on socioeconomic status and health. Hyattsville, MD: 2016.

[25] Caspersen CJ, Pereira MA, Curran KM. Changes in physical activity patterns in the United States, by sex and cross-sectional age. Med Sci Sport Exerc 2000;32:1601-9.

[26] Owen N, Sparling PB, Healy GN, et al. Sedentary behavior: emerging evidence for a new health risk. Mayo Clin Proc 2010;85:1138-41.

[27] Mansoubi M, Pearson N, Biddle SJH, et al. The relationship between sedentary behaviour and physical activity in adults: A systematic review. Prev Med (Baltim) 2014;69:28-35.

[28] Owen N, Healy GN, Matthews CE, et al. Too much sitting: The population-health science of sedentary behavior. Exerc Sport Sci Rev 2010;38:105-13.

[29] Katzmarzyk PT. Physical activity, sedentary behavior, and health: Paradigm paralysis or paradigm shift? Diabetes 2010;59:2717-25.

[30] Matthews CE, George SM, Moore SC, et al. Amount of time spent in sedentary behaviors and cause-specific mortality in US adults. Am J Clin Nutr 2012;95:437-45.

[31] Craft LL, Zderic TW, Gapstur SM, et al. Evidence that women meeting physical activity guidelines do not sit less: An observational inclinometry study. Int J Behav Nutr Phys Act 2012; 9: 122.

[32] Gopinath B, Hardy LL, Baur LA, et al. Physical activity and sedentary behaviors and health-related quality of life in adolescents. Pediatrics 2012;130:e167-74.

[33] Rebar AL, Duncan MJ, Short C, et al. Differences in healthrelated quality of life between three clusters of physical activity, sitting time, depression, anxiety, and stress. BMC Public Health 2014; 14: 1088.

[34] Goldfield GS, Cameron JD, Murray M, et al. Screen time is independently associated with health-related quality of life in overweight and obese adolescents. Acta Paediatr 2015; 104: e448-54.

[35] Loprinzi PD. Joint associations of objectively-measured sedentary behavior and physical activity with health-related quality of life. Prev Med Reports 2015; 2: 959-61.

[36] Martin SB, Morrow JR, Jackson AW, et al. Variables related to meeting the CDC/ACSM physical activity guidelines. Med Sci Sport Exerc 2000; 32: 2087-92.

[37] Craig CL, Marshall AL, Sjöström M, et al. International physical

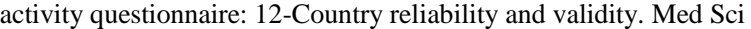
Sport Exerc 2003;35:1381-95.

[38] Centers for Disease Control and Prevention. How much physical activity do adults need? Available at: http://www.cdc.gov/physicalactivity/basics/adults/index.htm. AccessedJune 30, 2015.

[39] Morrow JR, Tucker JS, Jackson AW, et al. Meeting physical activity guidelines and health-related fitness in youth. Am J Prev Med 2013;44:439-44.

[40] Farren GL, Zhang T, Martin SB, et al. Factors related to meeting physical activity guidelines in active college students: A social cognitive perspective. J Am Coll Heal 2017;65:10-21.

[41] Rosenberg DE, Norman GJ, Wagner N, et al. Reliability and validity of the Sedentary Behavior Questionnaire (SBQ) for adults. J Phys Act Heal 2010;7:697-705.

[42] Deforche B, Van Dyck D, Deliens T, et al. Changes in weight, physical activity, sedentary behaviour and dietary intake during the transition to higher education: A prospective study. Int J Behav Nutr Phys Act 2015;12:16.

[43] Varni JW, Limbers CA. The PedsQL 4.0 Generic Core Scales Young Adult Version: feasibility, reliability and validity in a university student population. J Health Psychol 2009;14:611-22.

[44] Cohen JE, Cohen P, West SG, et al. Applied multiple regression/correlation analysis for the behavioral sciences. 3rd ed. New York, NY: Routledge; 2003.
[45] Zhang T, Xiang P, Gu X, et al. College students’ physical activity and health-related quality of life: An achievement goal perspective. Res Q Exerc Sport 2016;1367:1-9.

[46] Faul F, Erdfelder E, Buchner A, et al. Statistical power analyses using G*Power 3.1: Tests for correlation and regression analyses. Behav Res Methods 2009;41:1149-60.

[47] Enders CK. Applied missing data analysis. New York, NY: The Guilford Press; 2010.

[48] Hoaglin DC, Iglewicz B, Tukey JW. Performance of some resistant rules for outlier labeling. J Am Stat Assoc 1986;81:991-9.

[49] Kannan KS, Manoj K. Outlier detection in multivariate data. Appl Math Sci 2015;9:2317-24

[50] Henson RK. Multivariate normality: What is it and how is it assessed? Adv Soc Sci Methodol 1999;5:193-211.

[51] Hayes AF. PROCESS: A versatile computation tool for observed variable mediation, moderation, and conditional process modeling. The Ohio State University, 2012.

[52] Office of Institutional Reseach and Effectiveness. University of North Texas fact book 2014-2015. Denton, TX: 2015.

[53] Aiken LS, West SG. Multiple regression: Testing and interpreting interactions. Newbury Park, CA: SAGE Publications, Inc.; 1991.

[54] Little TD, Card NA, Bovaird JA, et al. Structural equation modeling of mediation and moderation with contextual factors. In: Little TD, Bovaird JA, Card NA, editors. Model. Context. Eff. Longitud. Stud. 1st ed., Mahwah, NJ: Lawrence Erlbaum Associates, Inc.; 2007, p. 207-30.

[55] Preacher KJ, Curran PJ, Bauer DJ. Computational tools for probing interactions in multiple linear regression, multilevel modeling, and latent curve analysis. J Educ Behav Stat 2006;31:437-48

[56] Fairclough SJ, Boddy LM, Mackintosh KA, et al. Weekday and weekend sedentary time and physical activity in differentially active children. J Sci Med Sport 2015;18:444-9.

[57] McVeigh JA, Winkler EAH, Howie EK, et al. Objectively measured patterns of sedentary time and physical activity in young adults of the Raine study cohort. Int J Behav Nutr Phys Act 2016;13:41

[58] Smith L, Hamer M, Ucci M, et al. Weekday and weekend patterns of objectively measured sitting, standing, and stepping in a sample of office-based workers: The active buildings study. BMC Public Health 2015;15:9.

[59] Fountaine CJ, Liguori GA, Mozumdar A, et al. Physical activity and screen time sedentary behaviors in college students. Int $\mathrm{J}$ Exerc Sci 2011;4:102-12.

[60] Pearson N, Ball K, Crawford D. Mediators of longitudinal associations between television viewing and eating behaviours in adolescents. Int J Behav Nutr Phys Act 2011;8:23.

[61] Buckworth J, Nigg C. Physical activity, exercise, and sedentary behavior in college students. J Am Coll Heal 2004;53:28-34.

[62] U.S. Department of Health and Human Services. 2008 Physical activity guildlines for Americans. Washington, DC: 2008.

[63] Prince SA, Adamo KB, Hamel ME, et al. A comparison of direct versus self-report measures for assessing physical activity in adults: A systematic review. Int J Behav Nutr Phys Act 2008; 5: 56-79.

[64] Mackay LM, Oliver M, Schofield GM. Demographic variations in discrepancies between objective and subjective measures of physical activity. Open J Prev Med 2011;1:13-9.

[65] Watkinson C, van Sluijs EMF, Sutton S, et al. Overestimation of physical activity level is associated with lower BMI: A crosssectional analysis. Int J Behav Nutr Phys Act 2010;7:1-9.

[66] Meyerson P, Tryon WW. Validating internet research: A test of the psychometric equivalence of internet and in-person samples. Behav Res Methods, Instruments, Comput 2003;35:614-20. 